\title{
A detection of the coarse water droplets in steam turbines
}

\author{
Ondřej Bartoš $^{1, a}$, Xiaoshu Cai ${ }^{2}$ and Michal Kolovratník ${ }^{1}$ \\ ${ }^{1}$ CTU in Prague, Department of Energy Engineering, Technická 4, Prague 6, 166 07, The Czech Rep. \\ ${ }^{2}$ University of Shanghai for Science and Technology, Jungong Road 516, Shanghai, China
}

\begin{abstract}
The aim of this paper is to introduce a novel method for the detection of coarse water droplets in a low pressure part of steam turbines. The photogrammetry method has been applied for the measurement of coarse droplets in the low-pressure part of a steam turbine. A new probe based on this measurement technique was developed and tested in the laboratory and in a steam turbine in the Počerady power-plant. The probe was equipped with state-of-the-art instrumentation. The paper contains results from laboratory tests and the first preliminary measurements in a steam turbine. Possible applications of this method have been examined.
\end{abstract}

\section{Introduction}

The liquid phase in the low pressure stages of steam turbines has a negative influence on the efficiency and reliability. Because of this, the study of the formation and evolution of water droplets in turbines is important. In the long term the authors of the paper are concerned with experimental research into the liquid phase structure in steam turbines [1,2].

Two populations of water droplets are present in steam turbines. Nucleation of droplets and their subsequent growth are responsible for the presence of the very fine droplets. Coarse droplets are formed by the disruption of the water films on the blade and casing surfaces in the low-pressure stages. The present knowledge of the coarse water flow field and the size distribution of the droplets in turbines is still insufficient. The coarse droplets are responsible for some unfavourable effects like additional energy losses and a reduction in the lifetime of the blades. A better knowledge about the formation of coarse droplets and their properties may bring future enhancements for the steam turbine.

A new adapted photogrammetric method was developed from classical photogrammetry. This technique was applied for determining the droplet size distribution function and for estimating the velocity field of the coarse droplets [3]. There are several advantages of the new method in comparison with previously used methods [4]. The photogrammetric method can be applied for measurements at low droplet number densities. This is the case in the low pressure stages of the steam turbine where the coarse droplets are distributed non-homogenously; the mean number density of coarse droplets is expected to be in the range 1 to 1000 droplets $/ \mathrm{cm}^{3}$. Another advantage of the photogrammetric method is its high spatial resolution. Unlike the light scattering and extinction techniques, the method should also provide information about the flow field of the droplets.

\section{Method}

Photogrammetry is a method for determining the object properties from a picture captured by a camera. The expected size of the coarse droplets in steam turbines is in a wide range of 1 to $500 \mu \mathrm{m}$. The theoretical resolution of the method is determined by the pixelpitch and the resolution of the objective lens. The size of the pixelpitch is in the range 2 to $6 \mu \mathrm{m}$ for common digital cameras. The magnification of the objective is limited by the minimum exposure time of the camera when a global shutter is used. Higher magnification can bring better resolution for smaller droplets, but the displacement of the droplet may then be bigger than the size of the observed volume when the picture is taken. The velocity of droplets can reach more than $200 \mathrm{~m} / \mathrm{s}$. The droplet velocities and the minimum exposure time define the size of the observed volume. The entire observed volume has to be imaged on the camera sensor and this requirement limits the magnification of the objective. This means that each droplet is represented on the picture as a line.

Figure 1 presents a schematic diagram of the measurement system and figure 2 shows an example of front side illumination of the droplet paths captured in the laboratory. The length of the path and the exposure time allows the computation the velocity of the droplet and determines the expected direction of the droplet's movement. But it is necessary to realise that the view of the motion is two dimensional. This means that one can see only the components of the velocity perpendicular to

\footnotetext{
${ }^{\mathrm{a}}$ Corresponding author: ondrej.bartos@fs.cvut.cz
} 
the axis of the camera. Behind the last low-pressure stage of a steam turbine it is expected that there will be a substantial radial component of droplet velocity. The depth of the observed volume is determined by the depth of field of the objective. A telecentric objective provides the advantage that the image size is independent of the distance from the objective. Calibration is required for the correct pixel/dimension ratio. A laboratory test with standard mono-sized particles and with the resolution target was in good agreement with the real size of the observed object.

\section{Laboratory tests}

All components of the probe were tested for the best performance on a laboratory setup. The camera (iDS GigE UI 5240CP1/1,8", $1280 \times 1024,10$ bits, $9 \mu \mathrm{s})$ was chosen because its size $(29 \times 29 \times 41 \mathrm{~mm})$ was small enough to mount inside the probe and the exposure time for the global shutter was $9 \mu \mathrm{s}$. During this time a droplet with a velocity of $200 \mathrm{~m} / \mathrm{s}$ moves $1.8 \mathrm{~mm}$. The size of the sensor is $6.784 \times 5.427 \mathrm{~mm}$ and the pixelpitch is $5.3 \mu \mathrm{m}$. The correct analysis of the droplet movement can be made if the entire path is captured on the sensor of the camera. Two types of telecentric objectives were tested.

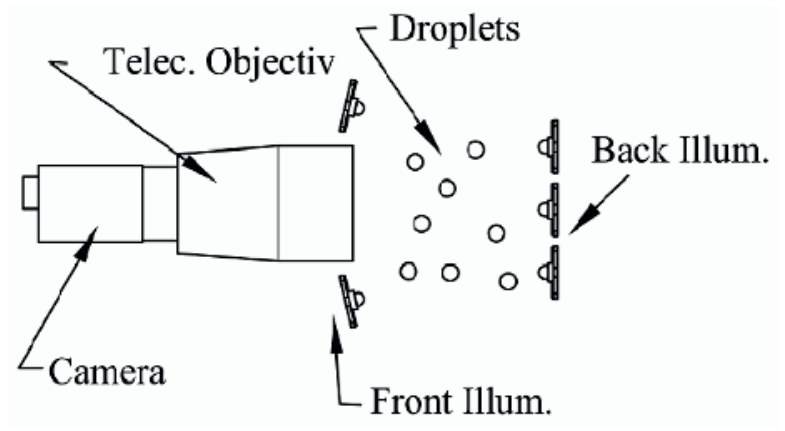

Figure 1. Schematic diagram of the measurement system.

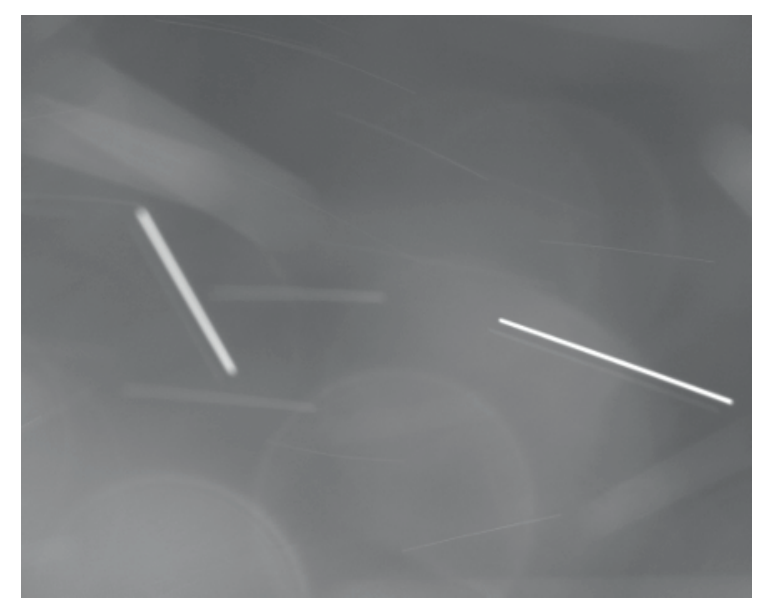

Figure 2. Front illumination of the droplet paths.

The objective lens was OPTO engineering TC 1224 (mag. $0.255 \mathrm{x}$, working distance $\sim 70 \mathrm{~mm}$, field depth 10 $\mathrm{mm}$ and theoretical resolution $16 \mu \mathrm{m}$ ). This objective was used for the laboratory testing with good results but the outer diameter exceeded the size limit given by the probe design. Moreover the resolution of the objective was not sufficient. The second objective was Can-Rill optics (mag. 1x, working distance $\sim 65 \mathrm{~mm}$, field depth $1.8 \mathrm{~mm}$ and theoretical resolution $10 \mu \mathrm{m}$ ). This objective was used in the probe.

Two possible positions of the light source were tested. figure 2 shows the front and figure 3 the back illumination, respectively. The advantage of frontal illumination is the brighter path of the droplets. The disadvantage is the technical limit of having a small homogeneous light source for the application mounted in the probe.

For the laboratory testing, a water nozzle with a high pressure pump as the source of the droplets was used. These tests were important for obtaining information about the behaviour of the optical components and for the choice of the optimal data processing method [5]. The data processing method can provide information about the size distribution of the droplets, their velocity and direction. For the test it was sufficient to use standard image enhancement and edge detection functions provided by Matlab.

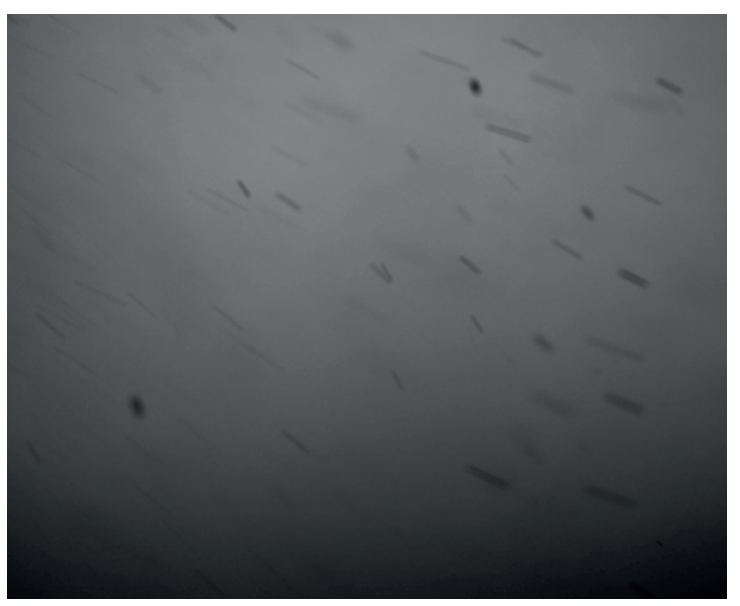

Figure 3. Droplet paths with back side illumination.

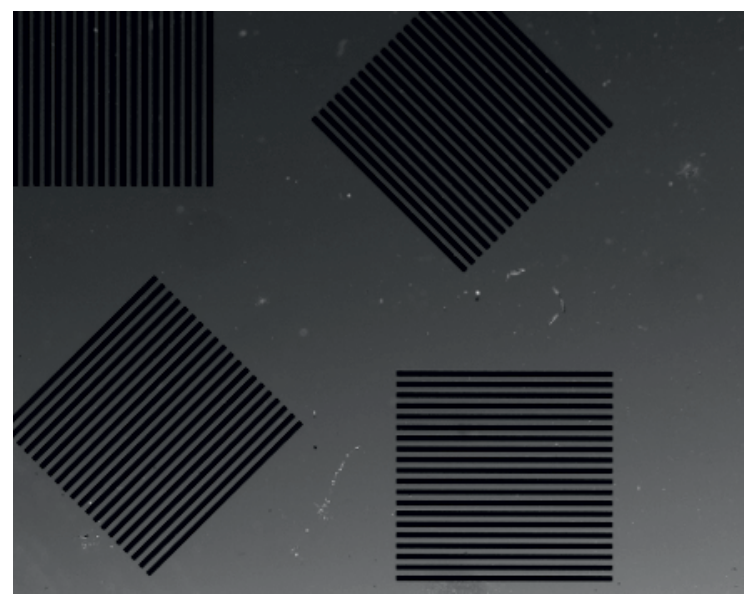

Figure 4. The resolution target with 20 strips per millimeter. 
Unlike light scattering, the photogrammetry method requires calibration. The calibration was carried out with the standard resolution target. This target was used for testing the spatial resolution of the new data processing method.

The figure 4 presents the calibration image with 20 stripes per a millimeter. The calibration target was also used for the determination of droplet's distance from the focus plane. This was done in a qualitative way by a variation of the light intensity I on the object edge.

$$
\alpha=d I / d x
$$

A position of the calibration target was moved out of the focal plane to yield the function. The figure 5 shows a dependence of the light intensity change on the edge of the object with respect the distance from the focal plane.

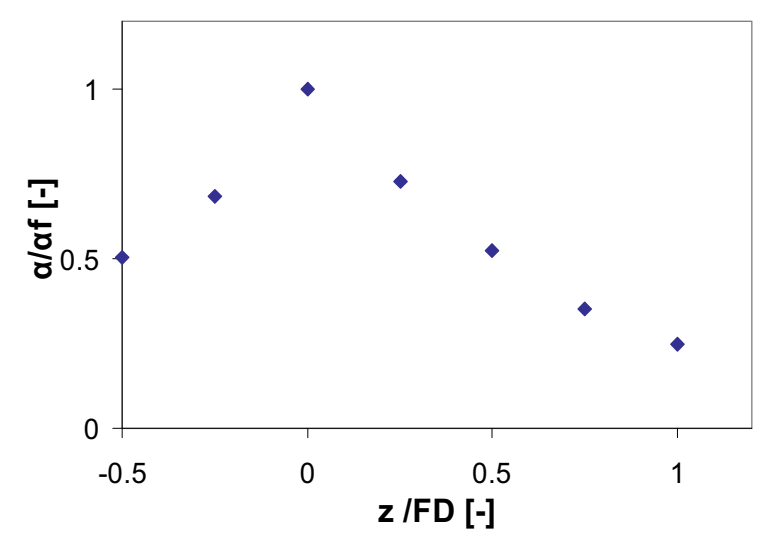

Figure 5. A light intensity drop on the edge of the object with vary the distance of the calibration target from the focal plane.

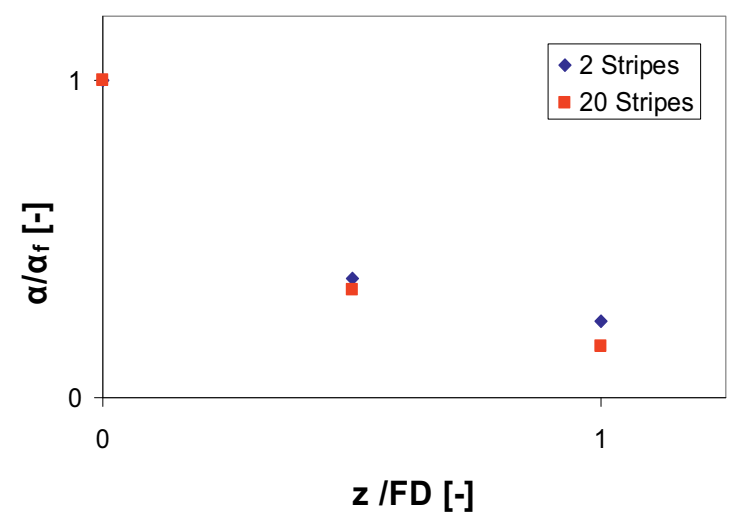

Figure 6. Dependence of with respect the distance normalized by the focal depth, $\alpha \mathrm{f}$ is the light intensity drop in the focal plane, FD is focal depth.

A dependence on the object size was verified with the calibration target with 2 and 20 stripes per millimeter. The normalized data are shown on the figure 6 , one can see that in the distance from the focal plane is the size dependence very small. This verification was done with optical set-up used in the probe; dependence on the optical components is obvious.

This approach can provide with a small uncertainty the decision if the particle is inside the observed volume or not. The example is shown on the figure 7 , the same particle is there in three different positions. The first image presents particle close to the focal plane, second is near the edge of the focal depth and third is out the observed volume.

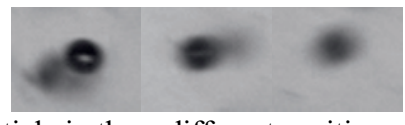

Figure 7. The particle in three different positions.

The figure 8 presents variation of the light intensity on the edge of the particle shown on figure 7 . In this case the glass particles were used.

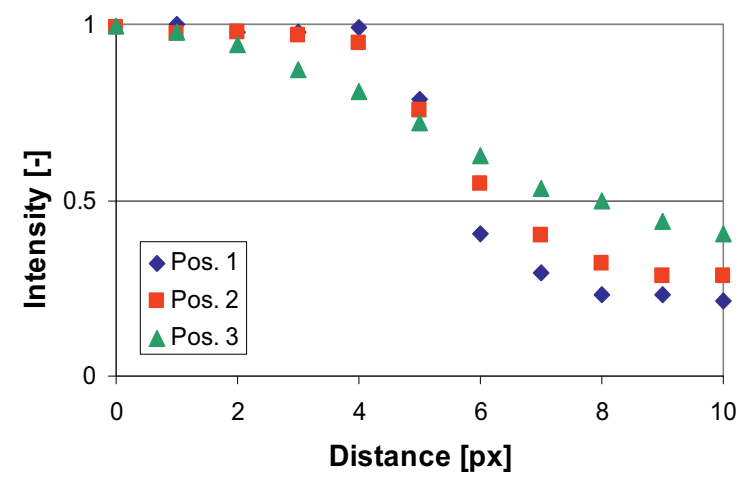

Figure 8. The light intensity profile of the glass particles edge.

At the first stage of the project it was difficult to predict the influence of the fine droplets on the measurements and on the data processing. Fine droplets can scatter light from the light source and cause blurriness of the captured image.

\section{Probe description}

The previous experience with measurement in steam turbines [6] was useful for the development of the probe. The probe is enclosed in a stainless steel tube $50 \times 3 \mathrm{~mm}$. The head of the probe slides out of the tubing at the measurement position. This system protects the probe head during the insertion of the probe through the casing of the turbine. The camera and the objective are in the head of the probe on one side of the test section. The LED light source is fixed on the other side. The size of the test section is determined by the two ribs, as shown on figure 9. The glass windows are purged by air. Previous experience has shown that the purging window is necessary to blow away the film of water deposited on the surface of the glass window. It is thought that the influence of the purging air curtain is negligible. When the probe is placed at the desired position in the main flow of the steam, the directions of the air and the steam 
flow are similar. The initial thickness of the air curtain is $0.8 \mathrm{~mm}$. A verification study showed that region of influence is of the order of a few millimetres above the glass surface. The theoretical resolution of the probe is given by the objective lens. In this case it was $10 \mu \mathrm{m}$.

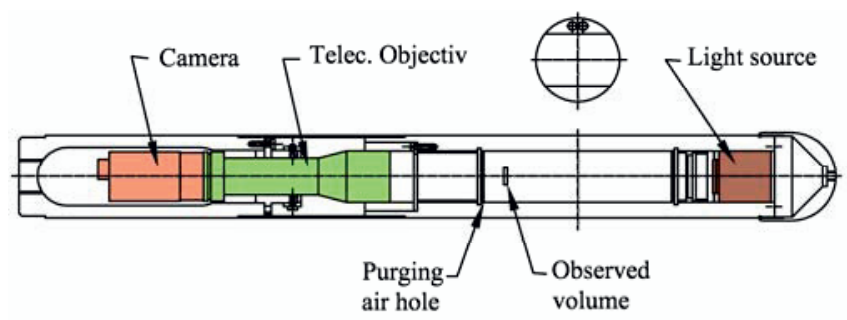

Figure 9. The probe's schema.

\section{Results and discussion}

The coarse droplets in steam turbines can appear in a wide range of diameters. In the past, some measurements with different probes have been carried out [4] to determine the size distribution of the droplets. The results show a range from 1 to $500 \mu \mathrm{m}$. This wide range is not easy to measure with one technique. The coarse droplets measurement technique described in this paper can be used above the resolution limit of the objective and camera. Under this limit the light scattering method can be used [7]. The light scattering technique cannot be used for larger droplets due to their small number density. The new probe is able to measure the small number density of droplets of this size.

A first verification measurement was performed in the low-pressure part of the $200 \mathrm{MW}$ steam turbine in the Počerady power plant, see figure 10 . The purpose of this measurement was to test the entire instrumentation system. The position of the probe was behind the last stage of the rotor. At this location the steam pressure was approximately $5 \mathrm{kPa}$ and the wetness was $6-8 \%$. The upper limit of droplet diameter can be estimated with an expected steam flow velocity (app. $200 \mathrm{~m} / \mathrm{s}$ ) up to 1000 $\mu \mathrm{m}$.

The probe was equipped with a needle as the visibility target; the tip of the needle was placed in the observation volume. The purpose was to prove that the observation volume was indeed visible to the camera through the layer of fine droplets (figure 11). The properties of the wet steam in this particular turbine have been measured in the past and determined mass flow rate per unit area of coarse droplets behind the last stage under design conditions can be about $0.1 \mathrm{~kg} \mathrm{~m}^{-2} \mathrm{~s}^{-1}$. The number density of coarse droplets with an expected unified diameter of $10 \mu \mathrm{m}$ is 120 droplets $\mathrm{cm}^{3}$. When the exposure time of the camera was $9 \mu$ s one coarse droplet could be seen every 4500 pictures. For that reason the aim of the first test was not to capture coarse droplet images in a sufficient amount to yield a distribution function but only to test the instrumentation and the technique, and to gain some experience with this measurement method.

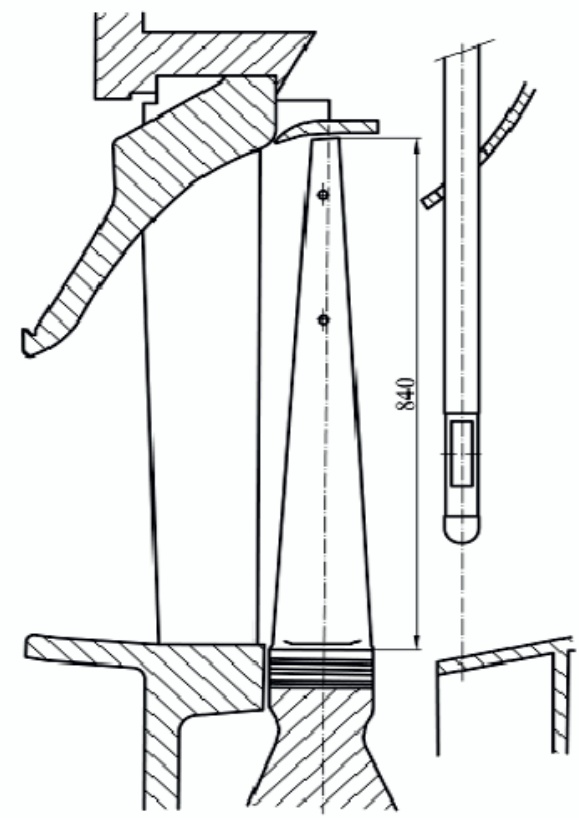

Figure 10. Measurement position in the steam turbine behind last stage.

\section{Conclusions}

A new measurement technique for the size distribution of coarse droplets was tested in a steam turbine. A first evaluation confirmed the ability of the method for this type of measurement. Although the probe design is simple, it uses state-of-the-art instrumentation. Future development of cameras, objectives, illumination and data processing will bring faster measurements with a higher resolution.

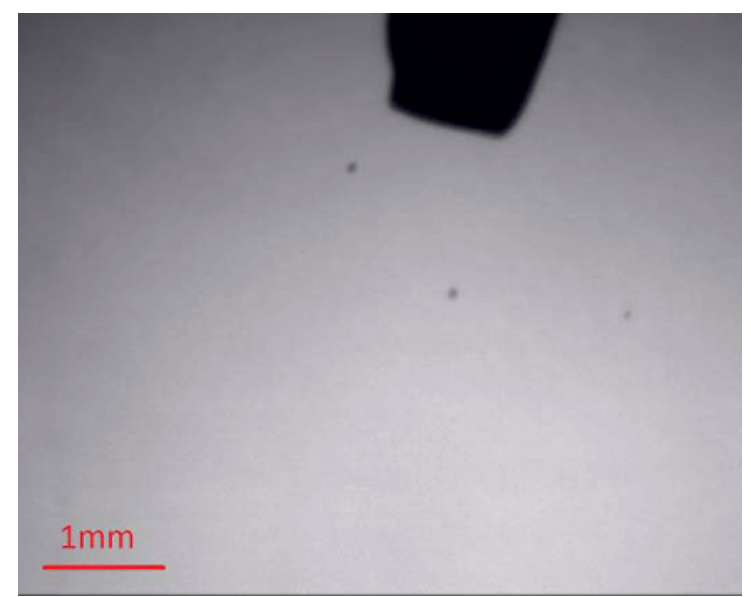

Figure 11. Secondary droplets $(\sim 60 \mu \mathrm{m})$ in vicinity of the needle.

The present design of the probe was successful but during the measurements in the steam turbine was difficult to distinguish between the coarse droplets produced by water shed from the blades and from the probe. A new design of probe is therefore under development. The main difference is in the application of 
the front illumination. The enhancement will allow the removal of the ribs.

The technique can also be used for the observation of the formation and disruption of liquid films inside steam turbines (figure 12).

Finally, it is concluded that the study of the coarse water in steam turbines can still provide new knowledge for improving the reliability and efficiency of the machines.

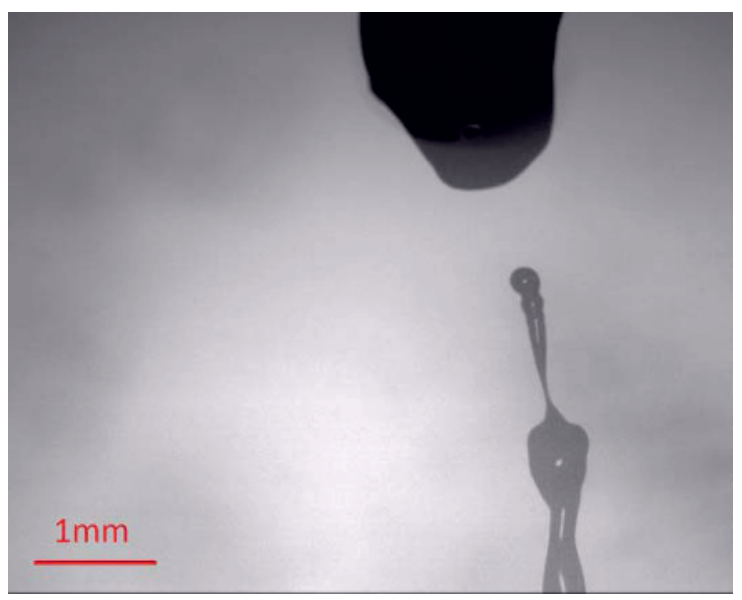

Figure 12. Water film disruption from the needle.

\section{Acknowledgement}

The work was supported by the Nature Science Foundation of China, grant number 51206112 and by Grant no. 101/11/1593 of the Grant Agency of the Czech Republic.

\section{References}

1. V. Petr, M. Kolovratník, Proc. 10th. Conf. on Steam and Gas Turbines, 213-218, (1994)

2. X. Cai,L. Wang, Y. Pan, X. Ouyan, J. Shen, J. Thermal Science. 10: 123-126, (2001)

3. H. Zhang, X. Cai, X. Wang, Z. Shang, T. Ning, Ann. Conf. Chinese Society of Engineering Thermalphysics on Multiphase Flow, 8, 20-25, (2007)

4. A. Kleitz, J.M. Dorey, Proceedings of The Institution of Mechanical Engineers Part C-journal of Mechanical Engineering Science, vol. 218, no. 8, pp. 811-842, (2004)

5. M.S. Nixon, A.S. Aguado, Feature extraction and image processing, 1st Ed., Newnes, UK, (2002)

6. M. Kolovratník O. Bartoš, 20th Jubilee Turbomachinery Workshop, IFFM Publishers, 32, 16, (2006)

7. O. Bartoš, M. Kolovratník, Proc. Int. Conf. Experimental Fluid Mechanics, 21-24, (2009) 